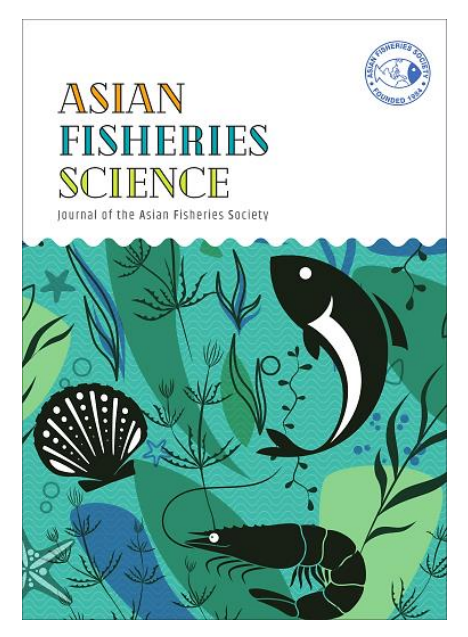

\title{
Bacterial Communities Associated With Penaeus vannamei Boone, 1931 Surface and Its Rearing Water in Biofloc Culture System
}

\author{
PALLAVI BALIGA, PUNEETH THADOORU GOOLAPPA, MALATHI SHEKAR* , S.K. GIRISHA, \\ RAMESH K.S., VILASINI UDYAVARA, M.N. VENUGOPAL \\ Department of Aquatic Animal Health Management, College of Fisheries, Karnataka Veterinary, Animal and Fisheries Sciences \\ University, Mangalore 575 002, Karnataka, India
}

*E-mail: malathishekar@rediffmail.com |Received: 03/12/2020; Accepted: 08/03/2021

(ㄷ) Asian Fisheries Society

ISSN: 0116-6514

E-ISSN: 2073-3720

https://doi.org/10.33997/j.afs.2021.34.1.004

\begin{abstract}
The biofloc system is an ecologically sustainable shrimp culture system. The conglomerates of beneficial bacteria, algae and protozoa in pond water serve as a water quality management system and as a feed additive to the shrimps. This study aimed to characterise the microbial communities associated with the biofloc pond water and the surface of Penaeus vannamei Boone, 1931, reared in it using the Illumina Miseq sequencing technology. The multiple alpha diversity measures indicated the shrimp surface samples to be richer in diversity than the pond water samples. Analysis of the bacterial community revealed that Proteobacteria, Bacteroidetes, Planctomycetes and Cyanobacteria formed the principal phyla. There was a shift in the relative abundance of bacterial communities at each time point. The operational taxonomic units (OTU) analyses revealed that $18.38 \%$ OTUs were shared by the pond water samples, the shrimp surface samples shared $29.35 \%$ at the three different time points. PICRUST analysis revealed that the bacterial communities in the biofloc rearing water, and shrimp surface, were likely involved in intensive microbial metabolism and core housekeeping functions. The information generated will help understand the bacterial community composition associated with optimal water quality and shrimp health in a biofloc culture system.
\end{abstract}

Keywords: 16s RNA, bacterial communities, Illumina Miseq, amplicon sequencing, super intensive farming

\section{Introduction}

Penaeus vannamei Boone, 1931, also known as the Pacific white shrimp, dominates the global aquaculture market as one of the highly farmed aquatic species (Fan et al., 2019). The conventional shrimp farming system is afflicted with several problems such as improper effluent disposal, escape of exotic species to the ecosystem and spread of diseases resulting in significant production losses (Rego et al., 2018). Recently, an alternative production system termed biofloc technology has been developed, the concept of which has been adopted by several shrimp farmers worldwide as a cost-effective method for increasing production and sustainable farming (Krummenauer et al., 2014).

The biofloc technology is based on nutrients recycling, high stocking densities and zero water exchange (Pilotto et al., 2018). The bioflocs are dominated by fungi, protozoa, zooplankton, microalgae, and heterotrophic bacteriawhich scavenge excess nitrogen and maintain a balance of nutrients in the water (Pilotto et al., 2018). The microbial flocs serve as food for the shrimps enhancing their immunity, growth performance, robustness and survival (Pilotto et al., 2018). Characterisation of the complex microbial communities associated with the bioflocs might help decipher the bacterial contribution towards optimal water quality and health of animals being cultured (Cardona et al., 2016). Very few studies have explored microbial communities' impact on shrimp health in a biofloc rearing system (Cardona et al., 2016; Pilotto et al., 2018; Deng et al., 2019). These studies have been based mainly on studying shrimp gut microbiota concerning the microbial community in biofloc system rearing waters. Shrimp share an intimate relationship with its rearing environment. The exoskeleton of shrimp serves as a host to several indigenous microbiota which acts primarily as a barrier against 
the entry of opportunistic pathogens (Vogan et al., 2008). Since no study exists on the microbiome diversity associated with $P$. vannamei surface in a biofloc system, this study attempts to understand the disease-free shrimp surface microbiome and its rearing water microbiome and the dynamic changes if any, during the culture period.

\section{Materials and Methods}

\section{Sample collection}

Penaeus vannamei samples cultured in a biofloc system were collected from a farm located at Mulki (Latitude: N 13063'422"; Longitude: E 74789'488") southwest coast Karnataka, India. The aquaculture pond at the farm was 0.1 hectare with water depth of $1.5 \mathrm{~m}$ and was lined with polyethylene sheet. The shrimp stocking density was $250 \mathrm{~m}^{-2}$. The pond was aerated $24 \mathrm{~h}$ using blow diffuser aerators of $80 \mathrm{hp}$ capacity. The average dissolved oxygen, $\mathrm{pH}$, temperature and salinity of the rearing pond water during the culture period were $6.0 \mathrm{mg} . \mathrm{L}^{-1}, 7.74,25^{\circ} \mathrm{C}$ and 22 ppt, respectively. The culture period lasted for 80 days. Throughout the culture period, the stocked shrimp and water was routinely being monitored for the presence of major shrimp pathogens such as white spot syndrome virus, infectious hypodermal and hematopoietic necrosis virus, monodon baculovirus, hepatopancreatic parvovirus, yellow head virus, Taura syndrome virus, infectious myonecrosis virus, Enterocytozoon hepatopenaei and Vibrios responsible for acute hepatopancreatic necrosis disease by polymerase chain reaction (PCR) using primers listed by OIE (Office International des Epizooties, 2003).

\section{Pond water sampling}

For each pond water sampling, $1 \mathrm{~L}$ of water sample was collected randomly from three different sites in duplicate from the same pond using sterile bottles. On-site, $200 \mathrm{~mL}$ of the collected water samples was drawn using sterile syringes and filtered by passing it through $0.45 \mu \mathrm{m}$ Whatman Cyclopore polycarbonate membranes (Sigma Aldrich cat\# WHA70604704) fitted on to a filter holder with Luer slip connector (Cole Parmer cat\# SC-06623-22). The filters were immediately stored in molecular-grade $100 \%$ ethanol and kept at $-20{ }^{\circ} \mathrm{C}$ until further use. The samples included in this study were pre-stocking water sample (BPS1) and three rearing water samples (BPW1, BPW2, BPW3) corresponding to $35^{\text {th }}, 55^{\text {th }}$ and $70^{\text {th }}$ day of stocking, respectively.

\section{Shrimp surface sampling}

To assess the shrimp surface microbiome, 10 shrimps were collected at each time point and kept in sterile water (autoclaved distilled water checked for sterility by plating on nutrient agar) for 20 min and the water processed in the same manner as for the pond water samples. The length and weight of shrimps were collected and analysed corresponding to $35^{\text {th }}$ (sample BS1), 55 th (sample BS2) and $70^{\text {th }}$ (sample BS3) day of stocking was $2.3 \mathrm{~cm} / 2 \mathrm{~g}, 9.2 \mathrm{~cm} / 8 \mathrm{~g}$ and $13.8 \mathrm{~cm} / 12 \mathrm{~g}$, respectively.

\section{DNA extraction, PCR amplification, purification and sequencing}

The filters corresponding to pond water and shrimp surface were subjected to DNA extraction and sequencing. The filters were vacuum-dried to remove ethanol followed by the addition of lysis buffer (30 mM Tris, 30 mM ethylene diamine tetra acetic acid (EDTA), $\mathrm{pH} 8$ ) to ensure complete lysis of the cells. The filters were stored in lysis buffer at $-80^{\circ} \mathrm{C}$ until the next use. For DNA extraction, the filters were thawed and incubated with lysozyme (50 mg. $\left.\mathrm{mL}^{-1}\right)$ at $37^{\circ} \mathrm{C}$ for 30 min. Following which, $10 \%$ SDS (sodium dodecyl sulfate) and proteinase $\mathrm{K}\left(20 \mathrm{mg} \cdot \mathrm{mL}^{-1}\right)$ was added and incubated at $55^{\circ} \mathrm{C}$ for one hour. The filter was then incubated with $5 \mathrm{M} \mathrm{NaCl}$ and $10 \%$ CTAB (cetyltrimethyl ammonium bromide) at $65{ }^{\circ} \mathrm{C}$ for $10 \mathrm{~min}$. The next step involved the addition of chloroform: isoamyl alcohol (24:1) followed by centrifugation at $14,000 \times g$ at $4^{\circ} \mathrm{C}$. The aqueous layer was collected in a fresh tube and the chloroform: isoamyl alcohol (24:1) wash was repeated twice, following which 0.1 volume of sodium acetate was added to the aqueous extract. DNA was precipitated by the addition of 0.7 volume of isopropanol to each tube at room temperature for 1-2 $\mathrm{h}$. The DNA pellet was obtained by centrifuging at $21,000 \times g$ for $30 \mathrm{~min}$ at room temperature. The pellets were washed with $70 \%$ ice-cold ethanol and air-dried and re-suspended in Tris EDTA buffer. The DNA samples were stored at $-20{ }^{\circ} \mathrm{C}$ until further use. Quantitation of dsDNA was performed using Qubit DNA HS quantitation assay kit (Thermo Scientific). The composition of bacterial communities was analysed by sequencing the hypervariable V3-V4 region of the 16SRNA using the primers $V 3 V 4 F$ : CCTACGGGNGGCWGCAG and V3V4R: GACTACHVGGG TATCTAATCC on the Illumina MiSeq platform $(2 \times 300$ bp) platform. The sequencing was outsourced to the DNA sequencing facility at Clevergene Pvt Ltd, Bengaluru, India.

\section{Data analysis}

Raw sequence reads were checked for their quality using FastQC and MultiǪC software. The generated reads were trimmed to remove the degenerate primers, adapter sequences and low -quality bases using the program Trim Galore (http://www .bioinformatics.babraham.ac.uk/projects/trim_galore

1). The paired sequence reads were aligned to form contigs using Mothur, an open-source software package (Schloss et al., 2009). The contig sequences shorter than 300 bp, duplicates, chimeric sequences and ambiguous nucleotides were further filtered out to obtain quality reads. The filtered contigs were processed and classified into taxonomical outlines and clustered into OTUs (Operational Taxonomic Unit) 
based on the GREENGENES v.13.8-99 database (DeSantis et al., 2006). PICRUSt (Langille et al., 2013) was used to predict gene family abundance. The rarefaction curve was generated using vegan $R$ package (Oksanen et al., 2018). Phyloseq R package was used for alpha diversity calculations. Principal coordinates analysis PCoA plot was generated using STAMP software (Parks et al., 2014). Alpha diversity was measured using seven different metrics (absolute number of observed OTUs, Chaol, ACE, Shannon, Simpson, InvSimpson, Fisher). The observed species index measures the count of unique OTUs in each sample. The species richness indices in the microbiome were estimated using Chaol and ACE indices (Chao, 1984; Colwell and Coddington, 1994). The "evenness" or homogeneity of the samples was estimated using Shannon; Fisher; Simpson and Inv Simpson indices (Jost, 2007). To evaluate the differences in OTU abundance between sample groups, the White's non-parametric t-test and Fisher's exact test was performed. A calculated value of $P<0.05$ was considered statistically significant.

\section{Results}

\section{Analysis of sequence reads}

In this study, a metagenomic analysis was undertaken to characterise the microbiome associated with $P$. vannamei surface and its rearing water cultivated using the biofloc system. Shrimp grown were healthy and showed no pathological signs of disease during the entire culture period. Sequencing of $\mathrm{V} 3-\mathrm{V} 4$ region of 16 srRNA generated $10,79,738$ reads were with average of 1,54,248 reads per sample (Table 1).

The rarefaction curves generated to evaluate the sufficiency of the sampling effort indicated that the shrimp surface had higher species richness than pond water. Most of the diversity was captured for the samples, but not all (Supplementary Fig. 1).
Alpha diversity measurement of richness and relative abundance of bacteria within the sample was measured using seven different metrics. Overall, the multiple alpha diversity measures indicated the shrimp surface samples to be richer in diversity than the pond water samples (Supplementary Table 1). Principal coordinates analysis was plotted to evaluate the beta diversity among samples (Supplementary Fig. 2). The PCoA plot represented a total variation of $55.7 \%$. The PCoA revealed that the microbial communities clustered into three distinct groups. The pond water and shrimp surface microbiome from the same culture stages clustered together. The microbial community from the pre-stocking water formed a separate group. Across all samples, $94 \%$ of the total reads obtained could be assigned into 48 phyla, 959 genera and 349 species while the remaining $6 \%$ grouped as unclassified.

\section{Microbial composition in the biofloc pond water}

The relative abundance graph showed the prestocking water (PS1) to be dominated by four major phyla such as Cyanobacteria (37\%), Bacteroidetes (20 $\%)$, Proteobacteria (17\%) and Planctomycetes (10\%). However, the relative abundances changed over the culture period.

The Proteobacteria and Bacteroidetes remained the most dominant phyla in the rearing water throughout the culture period with percentage relative abundances of $34 \%$ and $26 \%$, respectively. The Planctomycetes formed the third major phyla with a percentage relative abundance of $13 \%$. Cyanobacteria and Actinobacteria were the other major groups with relative abundance percentage greater than $5 \%$ (Fig. 1).

Among proteobacteria, the Gammaproteobacteria

Table1. Sequence reads, operational taxonomic units and their classification of the water and Penaeus vannamei samples from the biofloc pond.

\begin{tabular}{|c|c|c|c|c|c|c|c|c|c|}
\hline Sample & $\begin{array}{l}\text { Sample-ID } \\
\text { (DOC) }\end{array}$ & Reads & OTUs & Phyla & Class & Order & Family & Genus & $\begin{array}{l}\text { Bioproject } \\
\text { number } \\
\text { (GenBank) }\end{array}$ \\
\hline $\begin{array}{l}\text { Pre- } \\
\text { stocking } \\
\text { water }\end{array}$ & BPS1 & 167448 & 44787 & 34 & 87 & 172 & 268 & 429 & SRX7271112 \\
\hline \multirow{3}{*}{ Pond water } & BPW1 (35) & 123874 & 30094 & 34 & 83 & 154 & 240 & 344 & SRX7271113 \\
\hline & BPW2 (55) & 154676 & 35502 & 33 & 78 & 149 & 225 & 311 & SRX7271114 \\
\hline & BPW3 (70) & 178828 & 40298 & 40 & 96 & 173 & 279 & 411 & SRX7271115 \\
\hline \multirow{3}{*}{$\begin{array}{l}\text { Shrimp } \\
\text { surface }\end{array}$} & BS1 (35) & 157492 & 36936 & 38 & 103 & 200 & 328 & 510 & SRX7271116 \\
\hline & BS2 (55) & 133880 & 28207 & 37 & 94 & 186 & 301 & 468 & SRX7271117 \\
\hline & BS3 (70) & 163540 & 36002 & 37 & 99 & 189 & 314 & 506 & SRX7271118 \\
\hline
\end{tabular}




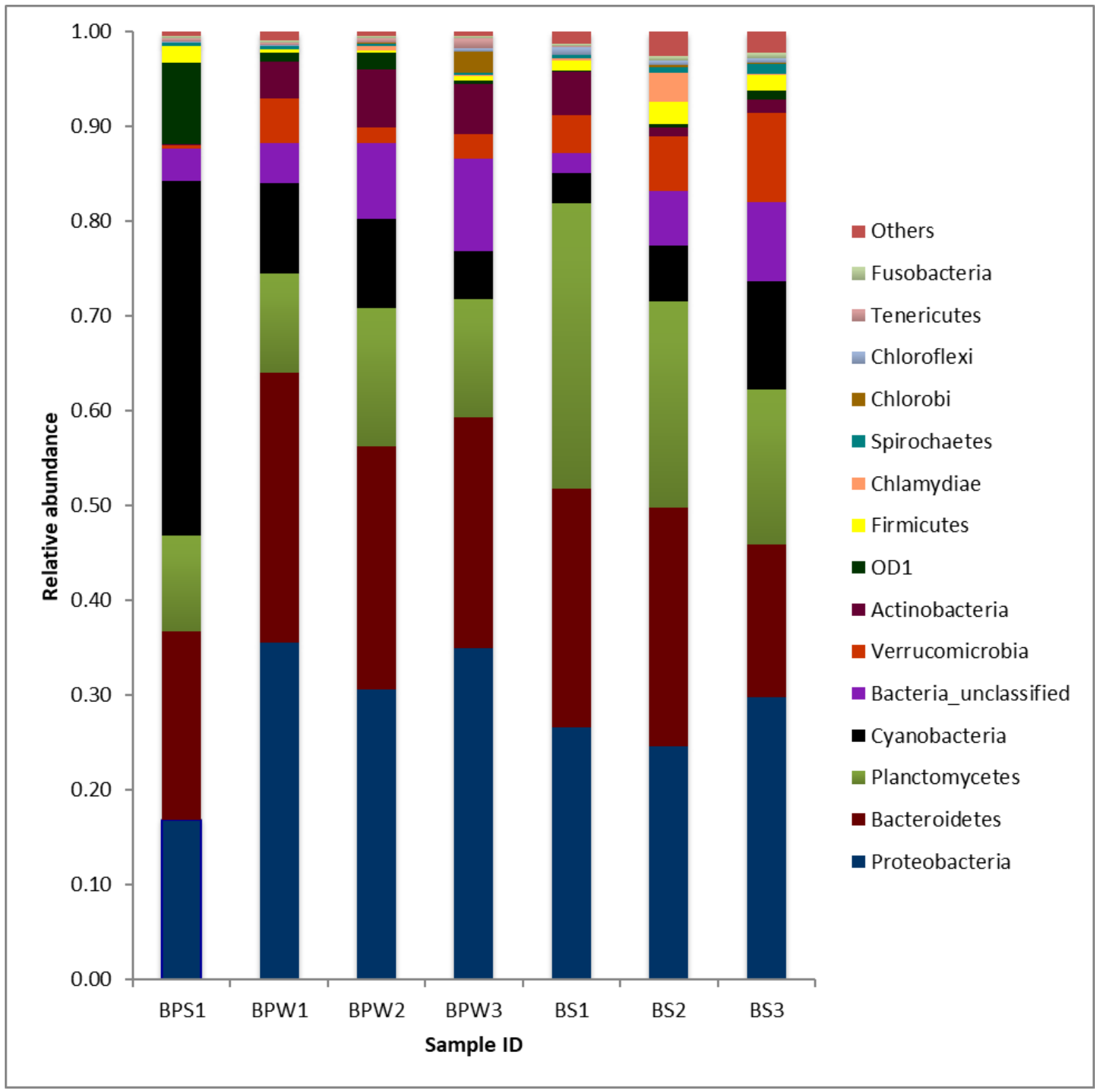

Fig. 1. Community bar plot analysis showing relative abundance of top 10 phyla BPS1- biofloc pre-stocking pond water; BPW1biofloc pond water ( $35 \mathrm{dps}$ ); BPW2- biofloc pond water ( $55 \mathrm{dps}$ ); BPW3- biofloc pond water (70 dps); BS1-BS3: biofloc Penaeus vannamei surface at 35,55 and 70 dps, respectively.

formed the largest class $(36 \%)$ followed by Alphaproteobacteria (32\%), Deltaproteobacteria (19 $\%$ ) and Betaproteobacteria (8\%). Among Gammaproteobacteria, Alteromonadaceae, OM60 and Pseudoalteromonadaceae were the major families enriched in pond water with mean relative frequencies of $1.41,1.21,0.23$, respectively $(P<0.05)$. Among Alphaproteobacteria, the families Rhodobacteraceae (4.77) and Rhodospirillaceae (1.76) were the major representatives $(P<0.05)$. The class Deltaproteobacteria was represented by Bacteriovoracaceae (0.11) $(P<0.05)$. Comamonadaceae (0.62) and Methylophilaceae (0.5) formed the dominant families among Betaproteobacteria in the pond water $(P<0.05)$.

Among the Bacteroidetes, Flavobacteriia formed the major group (42\%) followed by Saprospirae (26\%). The class Flavobacteriia was dominated by the families Flavobacteriaceae (8.63) and Cryomorphaceae (1.37) $(P<0.05)$. The class Saprospirae was dominated by the family Saprospiraceae $(4.75)(P<0.05)$.

Among Planctomycetes, the class Planctomycetia $(81 \%)$ formed the largest group with the family Pirellulaceae (8.68) $(P<0.05)$.

The class Synechococcophycideae (21 \%) and Oscillatoriophycideae (17 \%) were the major representatives among the phylum Cyanobacteria. Synechococcaceae $(1.54 \%)$ was the major representative from the class Synechococcophycideae and Phormidiaceae (0.95\%) from the class Oscillatoriophycideae $(P<0.05)$. 


\section{Microbial composition on shrimp surface}

The shrimp surface microbiome analysis showed dominance by three phyla; Proteobacteria, Bacteroidetes and Planctomycetes with relative abundance levels $>22 \%$ throughout the culture period. Cyanobacteria and Verrucomicrobia showed relative abundances $>3 \%$ on the shrimp surface (Fig. 1).

Distribution patterns of the Rhodobacteraceae on the shrimp surface were reflective of their distribution in pond water. Similar trends were noted for Rhodospirillaceae as well. Among Betaproteobacteria, the levels of Alcaligenaceae decreased 55 days onwards. Opposite trends were noted for Comamonadaceae and Methylophilaceae whose levels increased 55 days onwards. Bacteriovoracaceae, Bdellovibrionaceae, Nannocystaceae, Polyangiaceae was the major members of the Deltaproteobacteria on the shrimp surface. Alteromonadaceae,
Pseudoalteromonadaceae, Chromatiaceae Moraxellaceae, and Vibrionaceae were the major groups from Gammaproteobacteria that were enriched on the shrimp surface. The levels of Chitinophagaceae and Saprospiraceae were reflective of the levels in pond water and followed a similar trend. Bacteroidaceae, Prevotellaceae and Cyclobacteriaceae levels decreased 55 days onwards. Marinilabiaceae, Cytophagaceae, Flammeovirgaceae, Cryomorphaceae, Flavobacteriaceae were the other dominant members from the Phylum Bacteroidetes. Pirellulaceae and Planctomycetaceae dominated the shrimp surface among the Phylum Planctomycetes. Phormidiaceae, Synechococcaceae, Pseudanabaenaceae was the cyanobacterial members dominating the shrimp surface. From the Phylum Verrucomicrobia, the relative abundance levels of Opitutaceae decreased after 35 days, whereas the levels of Puniceicoccaceae increased after 35 days. Verrucomicrobiaceae levels remained stable on the shrimp surface (Table 2).

Table 2. The relative abundance percentage of bacterial operational taxonomic units at class level in the rearing pond water and Penaeus vannamei surface samples at different stages of growth in a biofloc culture system.

\begin{tabular}{|c|c|c|c|c|c|c|c|}
\hline \multirow{2}{*}{ Taxon } & \multicolumn{4}{|c|}{ Pond water samples } & \multicolumn{3}{|c|}{ Shrimp samples } \\
\hline & BPS1 & BPW1 & BPW2 & BPW3 & BS1 & BS2 & BS3 \\
\hline \multicolumn{8}{|c|}{ Phylum - Proteobacteria; Class-Alphaproteobacteria, Family: } \\
\hline Rhodobacteraceae & 63 & 74 & 33 & 38 & 66 & 42 & 51 \\
\hline Erythrobacteraceae & 12 & 1 & 0 & 0 & 3 & 3 & 2 \\
\hline Hyphomonadaceae & 7 & 4 & 1 & 2 & 5 & 2 & 3 \\
\hline Pelagibacteraceae & 0 & 0 & 21 & 20 & 0 & 1 & 2 \\
\hline Rhodospirillaceae & 4 & 10 & 22 & 16 & 3 & 16 & 5 \\
\hline Sphingomonadaceae & 0 & 6 & 0 & 0 & 2 & 0 & 0 \\
\hline Phyllobacteriaceae & 0 & 0 & 0 & 3 & 2 & 1 & 2 \\
\hline Hyphomicrobiaceae & 1 & 0 & 0 & 0 & 4 & 2 & 2 \\
\hline Others & 8 & 0 & 3 & 2 & 4 & 5 & 10 \\
\hline Unclassified & 6 & 4 & 20 & 18 & 10 & 27 & 24 \\
\hline \multicolumn{8}{|c|}{ Phylum - Proteobacteria; Class-Betaproteobacteria, Family: } \\
\hline Alcaligenaceae & 60 & 61 & 0 & 0 & 94 & 4 & 5 \\
\hline Comamonadaceae & 19 & 33 & 2 & 2 & 4 & 32 & 15 \\
\hline Methylophilaceae & 17 & 1 & 1 & 2 & 1 & 5 & 49 \\
\hline Others & 4 & 4 & 31 & 64 & 1 & 54 & 29 \\
\hline Unclassified & 0 & 1 & 66 & 31 & 0 & 4 & 1 \\
\hline \multicolumn{8}{|c|}{ Phylum - Proteobacteria; Class-Deltaproteobacteria, Family: } \\
\hline Bacteriovoracaceae & 21 & 1 & 1 & 3 & 3 & 15 & 9 \\
\hline Bdellovibrionaceae & 6 & 0 & 1 & 3 & 2 & 21 & 5 \\
\hline Desulfobacteraceae & 3 & 1 & 0 & 0 & 15 & 1 & 2 \\
\hline Desulfomicrobiaceae & 18 & 0 & 0 & 0 & 7 & 3 & 1 \\
\hline
\end{tabular}




\begin{tabular}{|c|c|c|c|c|c|c|c|}
\hline \multirow{2}{*}{ Taxon } & \multicolumn{4}{|c|}{ Pond water samples } & \multicolumn{3}{|c|}{ Shrimp samples } \\
\hline & BPS1 & BPW1 & BPW2 & BPW3 & BS1 & BS2 & BS3 \\
\hline Nannocystaceae & 5 & 0 & 0 & 0 & 15 & 11 & 3 \\
\hline Polyangiaceae & 5 & 1 & 1 & 0 & 10 & 18 & 5 \\
\hline Others & 29 & 79 & 60 & 85 & 8 & 5 & 4 \\
\hline unclassified & 14 & 18 & 36 & 8 & 40 & 25 & 71 \\
\hline \multicolumn{8}{|c|}{ Phylum - Proteobacteria; Class-Gammaproteobacteria, Family: } \\
\hline Alteromonadaceae & 22 & 4 & 7 & 4 & 7 & 10 & 3 \\
\hline Pseudoalteromonadaceae & 4 & 5 & 4 & 5 & 4 & 15 & 17 \\
\hline Xanthomonadaceae & 0 & 0 & 5 & 0 & 5 & 1 & 0 \\
\hline Oceanospirillaceae & 0 & 0 & 2 & 0 & 2 & 2 & 0 \\
\hline Francisellaceae & 1 & 2 & 0 & 2 & 0 & 3 & 2 \\
\hline Chromatiaceae & 4 & 0 & 12 & 0 & 12 & 3 & 0 \\
\hline Moraxellaceae & 0 & 1 & 10 & 1 & 10 & 4 & 8 \\
\hline Enterobacteriaceae & 2 & 2 & 7 & 2 & 7 & 1 & 0 \\
\hline Vibrionaceae & 1 & 2 & 1 & 2 & 1 & 8 & 19 \\
\hline Pseudomonadaceae & 0 & 0 & 1 & 0 & 1 & 6 & 2 \\
\hline Aeromonadaceae & 0 & 0 & 3 & 0 & 3 & 0 & 0 \\
\hline OM60 & 22 & 8 & 4 & 8 & 4 & 3 & 1 \\
\hline Others & 4 & 9 & 6 & 9 & 6 & 5 & 6 \\
\hline Unclassified & 40 & 67 & 38 & 67 & 38 & 39 & 39 \\
\hline \multicolumn{8}{|c|}{ Phylum - Bacteroidetes; Class-Saprospirae, Family: } \\
\hline Chitinophagaceae & 4 & 97 & 0 & 17 & 61 & 4 & 5 \\
\hline Saprospiraceae & 96 & 2 & 98 & 81 & 38 & 95 & 94 \\
\hline Unclassified & 0 & 0 & 2 & 2 & 1 & 1 & 1 \\
\hline \multicolumn{8}{|c|}{ Phylum - Bacteroidetes; Class-Bacteroidia, Family: } \\
\hline Bacteroidaceae & 27 & 19 & 31 & 45 & 30 & 17 & 11 \\
\hline Marinilabiaceae & 4 & 19 & 19 & 9 & 15 & 22 & 13 \\
\hline Prevotellaceae & 14 & 6 & 8 & 18 & 10 & 5 & 3 \\
\hline Others & 39 & 29 & 31 & 4 & 24 & 49 & 68 \\
\hline Unclassified & 16 & 27 & 12 & 24 & 20 & 7 & 5 \\
\hline \multicolumn{8}{|c|}{ Phylum - Bacteroidetes; Class-Cytophagia, Family: } \\
\hline Cyclobacteriaceae & 55 & 96 & 28 & 5 & 93 & 10 & 0 \\
\hline Cytophagaceae & 0 & 1 & 4 & 3 & 0 & 83 & 36 \\
\hline Flammeovirgaceae & 6 & 1 & 40 & 57 & 5 & 2 & 42 \\
\hline Others & 0 & 0 & 0 & 0 & 0 & 0 & 0 \\
\hline Unclassified & 39 & 2 & 28 & 35 & 1 & 5 & 21 \\
\hline \multicolumn{8}{|c|}{ Phylum - Bacteroidetes; Class-Flavobacteriia, Family: } \\
\hline Cryomorphaceae & 7 & 4 & 25 & 7 & 1 & 15 & 18 \\
\hline Flavobacteriaceae & 67 & 95 & 64 & 45 & 98 & 76 & 77 \\
\hline Others & 0 & 0 & 0 & 0 & 0 & 0 & 0 \\
\hline Unclassified & 25 & 1 & 10 & 48 & 1 & 9 & 4 \\
\hline \multicolumn{8}{|c|}{ Phylum - Planctomycetes; Class-Phycisphaerae, Family: } \\
\hline Phycisphaeraceae & 1 & 1 & 1 & 1 & 2 & 1 & 24 \\
\hline
\end{tabular}




\begin{tabular}{|c|c|c|c|c|c|c|c|}
\hline \multirow{2}{*}{ Taxon } & \multicolumn{4}{|c|}{ Pond water samples } & \multicolumn{3}{|c|}{ Shrimp samples } \\
\hline & BPS1 & BPW1 & BPW2 & BPW3 & BS1 & BS2 & BS3 \\
\hline Unclassified & 99 & 99 & 99 & 99 & 98 & 99 & 76 \\
\hline \multicolumn{8}{|c|}{ Phylum - Planctomycetes; Class-Planctomycetia, Family: } \\
\hline Pirellulaceae & 99 & 98 & 79 & 84 & 94 & 61 & 58 \\
\hline Planctomycetaceae & 0 & 1 & 20 & 15 & 3 & 36 & 38 \\
\hline Isosphaeraceae & 0 & 0 & 0 & 0 & 1 & 1 & 0 \\
\hline Gemmataceae & 0 & 1 & 0 & 0 & 2 & 1 & 0 \\
\hline Unclassified & 0 & 0 & 1 & 1 & 0 & 1 & 3 \\
\hline \multicolumn{8}{|c|}{ Phylum - Cyanobacteria; Class-Chloroplast, Family: } \\
\hline Chlamydomonadaceae & 0 & 0 & 0 & 0 & 4 & 1 & 0 \\
\hline Mamiellaceae & 0 & 0 & 0 & 2 & 0 & 0 & 0 \\
\hline unclassified & 100 & 100 & 100 & 98 & 96 & 99 & 100 \\
\hline \multicolumn{8}{|c|}{ Phylum - Cyanobacteria; Class-Oscillatoriophycideae, Family: } \\
\hline Phormidiaceae & 3 & 7 & 92 & 94 & 4 & 91 & 95 \\
\hline Others & 93 & 41 & 0 & 2 & 76 & 5 & 1 \\
\hline Unclassified & 5 & 52 & 8 & 4 & 21 & 4 & 4 \\
\hline \multicolumn{8}{|c|}{ Phylum - Cyanobacteria; Class-Synechococcophycideae, Family: } \\
\hline Synechococcaceae & 69 & 88 & 91 & 91 & 28 & 54 & 24 \\
\hline Pseudanabaenaceae & 29 & 6 & 0 & 3 & 68 & 38 & 76 \\
\hline Unclassified & 2 & 7 & 9 & 6 & 4 & 7 & 0 \\
\hline \multicolumn{8}{|c|}{ Phylum - Verrucomicrobia; Class- Spartobacteria, Family: } \\
\hline Chthoniobacteraceae & 88 & 100 & 100 & 100 & 100 & 100 & 50 \\
\hline Unclassified & 13 & 0 & 0 & 0 & 0 & 0 & 50 \\
\hline \multicolumn{8}{|c|}{ Phylum - Verrucomicrobia; Class-Opitutae, Family: } \\
\hline Opitutaceae & 19 & 99 & 86 & 0 & 82 & 0 & 1 \\
\hline Puniceicoccaceae & 75 & 0 & 5 & 62 & 2 & 73 & 71 \\
\hline Others & 6 & 0 & 5 & 23 & 1 & 9 & 20 \\
\hline Unclassified & 0 & 1 & 4 & 15 & 15 & 17 & 8 \\
\hline \multicolumn{8}{|c|}{ Phylum - Verrucomicrobia; Class-Verrucomicrobiae, Family: } \\
\hline Verrucomicrobiaceae & 100 & 100 & 100 & 100 & 100 & 100 & 100 \\
\hline \multicolumn{8}{|c|}{ Phylum - Actinobacteria; Class-Acidimicrobiia, Family: } \\
\hline C111 & 100 & 96 & 100 & 85 & 94 & 87 & 77 \\
\hline Others & 0 & 0 & 0 & 13 & 2 & 10 & 17 \\
\hline Unclassified & 0 & 4 & 0 & 2 & 4 & 3 & 6 \\
\hline \multicolumn{8}{|c|}{ Phylum - Actinobacteria; Class-Actinobacteria, Family: } \\
\hline Microbacteriaceae & 79 & 62 & 81 & 95 & 0 & 11 & 26 \\
\hline Micrococcaceae & 0 & 0 & 0 & 0 & 0 & 2 & 51 \\
\hline Others & 13 & 1 & 0 & 0 & 3 & 23 & 11 \\
\hline Unclassified & 8 & 36 & 19 & 5 & 96 & 64 & 12 \\
\hline \multicolumn{8}{|c|}{ Phylum - Actinobacteria; Class-Nitriliruptoria, Family: } \\
\hline Nitriliruptoraceae & 0 & 100 & 100 & 100 & 0 & 0 & 100 \\
\hline \multicolumn{8}{|l|}{ Phylum - OD1; Family: } \\
\hline Unclassified & 100 & 100 & 100 & 100 & 100 & 100 & 100 \\
\hline
\end{tabular}




\begin{tabular}{|c|c|c|c|c|c|c|c|}
\hline \multirow{2}{*}{ Taxon } & \multicolumn{4}{|c|}{ Pond water samples } & \multicolumn{3}{|c|}{ Shrimp samples } \\
\hline & BPS1 & BPW1 & BPW2 & BPW3 & BS1 & BS2 & BS3 \\
\hline \multicolumn{8}{|c|}{ Phylum - Firmicutes; Class-Bacilli, Family: } \\
\hline Exiguobacteraceae & 1 & 0 & 2 & 0 & 5 & 1 & 47 \\
\hline Bacillaceae & 13 & 0 & 0 & 5 & 9 & 64 & 10 \\
\hline Staphylococcaceae & 44 & 5 & 5 & 22 & 4 & 17 & 2 \\
\hline Others & 25 & 53 & 61 & 36 & 76 & 7 & 13 \\
\hline Unclassified & 17 & 42 & 32 & 37 & 5 & 10 & 28 \\
\hline \multicolumn{8}{|c|}{ Phylum - Firmicutes; Class-Clostridia, Family: } \\
\hline Acidaminobacteraceae & 27 & 9 & 13 & 2 & 0 & 7 & 27 \\
\hline Clostridiaceae & 35 & 5 & 0 & 5 & 2 & 2 & 1 \\
\hline Lachnospiraceae & 4 & 23 & 8 & 25 & 25 & 28 & 15 \\
\hline Ruminococcaceae & 14 & 18 & 17 & 20 & 30 & 26 & 7 \\
\hline Veillonellaceae & 2 & 14 & 8 & 15 & 33 & 12 & 4 \\
\hline Others & 9 & 5 & 0 & 15 & 3 & 7 & 21 \\
\hline Unclassified & 8 & 27 & 54 & 16 & 6 & 19 & 24 \\
\hline
\end{tabular}

\section{Unique common operational taxonomic units (OTUs)}

A Venn diagram was constructed to visualise the overlap and difference in microbial communities in the pre-stocking water (BPS1), biofloc rearing water (BPW1, BPW2, BPW3) and the shrimp surfaces (BS1, BS2, BS3) in different combinations. Results showed 152 OTUs out of 827 OTUs (18.38\%) were shared by the pond water samples regardless of their abundance levels throughout the sampling period (Fig. 2a). These OTUs probably formed the core microbiome in the biofloc pond water. Similarly, a comparison of the OTUs on the shrimp surface revealed that 287 OTUs were overlapping $(29.35 \%)$ out of the total 978 OTUs during the three samplings (Fig. 2b). These OTUs probably formed the core microbiome on the shrimp surface. Furthermore, the pond water and the shrimp surface shared $308(43.69 \%), 285(45.24 \%)$ and 351 $(47.9 \%)$ OTUs during three different time points (Figs. $2 c, d$, and e). This indicates that the pond water has a significant influence on the shrimp surface microbiome.

Genus Nitriliruptor (Otu0533), Ochrobactrum pseudintermedium (0tu0796) $(P<0.05)$ were the dominant bacterial groups that were unique to the pond water. Family Intrasporangiaceae (Otu0350), order Myxococcales (Otu0224), Psychrobacter meningitides (Otu0210), genus Thiothrix (Otu0489), Thermoanaerovibrio (Otu0408) were the dominant bacterial groups $(P<0.05)$ that were unique to the shrimp surface.

Investigation of microbial communities to predict metagenomic function using PICRUSt showed the dominant functional categories belonging to pathways involving carbohydrate and amino acid metabolism and core housekeeping functions in both shrimp surface and water. There was no functional difference between the shrimp and water samples during the sampling period.

\section{Discussion}

This study explored the bacterial population's diversity and dynamics on the shrimp surface and its rearing water in the biofloc culture system. Based on the diversity indices, the shrimp surfaces harboured more diverse phylotypes compared to the pond water. Proteobacteria was the overall dominant phylum across all samples sequenced in this study, followed by Bacteroidetes, Planctomycetes and Cyanobacteria. The trends in the phylum distribution observed in this study were similar to that reported earlier for a biofloc system (Cardona et al., 2016, Huerta-Rábago et al., 2019). The Proteobacteria is the most predominant phylum associated with shrimp and coastal areas and aquaculture ponds (Dai et al., 2018) and engage in nitrogen cycling and mineralisation of organic compounds (Deng et al., 2019).

Rhodobacteraceae are often associated with biofilms and are the first to colonise the aquatic surfaces (Rathgeber et al., 2005). The high concentration of suspended solids in the biofloc system serves as growth sites for this bacterial family. Furthermore, it has been postulated that the members of this group limit the survival of pathogenic vibrios by their antagonistic activity (Pilotto et al., 2018). 
a

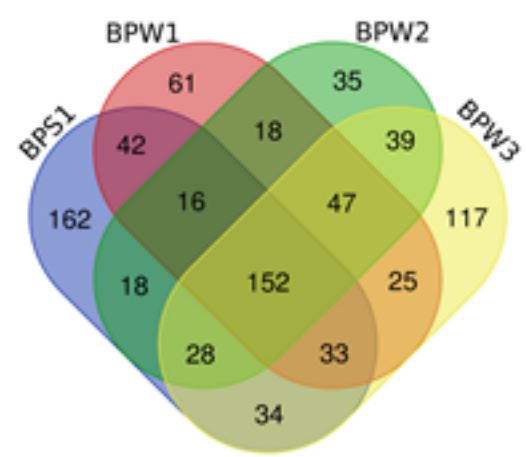

b

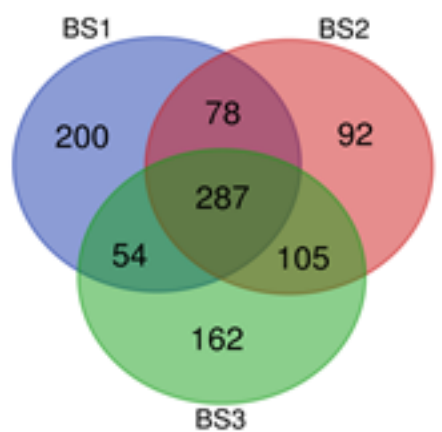

e

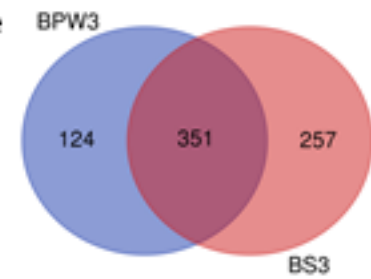

Fig. 2. Venn diagram showing the unique and shared OTUs among a) The pre-stocking and pond water samples b) the shrimp surface samples c, d,e) pond water and shrimp surface samples at 35, 55 and 70 days post stocking. BPS1- biofloc pre-stocking pond water; BPW1-biofloc pond water (35 dps); BPW2- biofloc pond water ( $55 \mathrm{dps}$ ); BPW3-biofloc pond water (70 dps); BS1 BS3: biofloc shrimp surface at 35,55 and $70 \mathrm{dps}$, respectively.

The Bacteroidetes constitute a large heterotrophic bacterioplankton community and are frequently found colonising macroscopic particles (Pilotto et al., 2018). The heterotrophic Flavobacterium spp. is abundant in aquatic ecosystems and is believed to play a specialised role in uptake and degradation of the organic matter such as proteins and complex polysaccharides in these environments (Trappen et al. 2003). The Flavobacteriales have been regarded as the indicators of healthy shrimp (Zheng et al., 2017). The water from the biofloc ponds is rich in organic matter and favour the growth of bacteria that use organic matter and nitrogenous compounds for growth (Pilotto et al., 2018).

In this study, a decrease in the level of Cyanobacteria was observed in the pond water relative to prestocking levels. Earlier studies have reported similar observations and suggested that the higher Cyanobacteria levels in the initial period could be related to the increased availability of organic carbon and higher light penetration while biofloc did not reach high concentrations (Emerenciano et al., 2013). Alternatively, a dip in cyanobacterial levels has been attributed to predation or substrate competition with small bacteria and other microorganisms (Emerenciano et al., 2013).

Planctomycetes have been recorded as one of the dominant phyla associated with shrimp culture ecosystems (Hou et al., 2017). Earlier studies have shown that such Planctomycetes prefer a biofilm lifestyle, attaching to surfaces in aquatic ecosystems, and their genetic makeup suggests a role in carbon turnover with the ability to degrade complex polysaccharides (Bengtsson et al., 2010).

Overall, the pond water shared lesser OTUs when compared to the shrimp surface. The unshared OTUs reflect the temporal shifts of the bacterioplankton community in pond water and the shrimp surface. The OTUs that form the core microbiota might play important roles in the microbiome's function and stability in the biofloc system. Even though lesser OTUs were shared among the samples, PICRUST analysis revealed no functional difference among the groups. The analysis revealed that the bacterial community was likely involved in microbial metabolism and core housekeeping functions.

The sequencing analysis indicated a predominance of the Proteobacteria community across samples. In earlier studies, KEGG pathway analysis for the phylum Proteobacteria has assigned functional roles such as carbohydrate, amino acid and nucleotide metabolism (Vikram et al., 2015).

The pond water and the shrimp surface shared $>43 \%$ of the OTUs, indicating the influence of the bioflocs on the surface microbiota of shrimp. Zero water exchange and high oxygenation rates stimulate the growth of heterotrophic bacteria, which positively influence shrimp growth and health status.

The microorganisms present in the biofloc water counter the pathogenic bacteria by competing for substrate and nutrients, producing inhibitory compounds, and interfering in the bacterial quorum- 
sensing (Pilotto et al., 2018). This was reflected by the good health status and $>80 \%$ survival rates of shrimps in this study.

\section{Conclusion}

The present study explored the diversity of bacterial communities in a biofloc culture system by applying 16S RNA next-generation sequencing approach. The findings in this study demonstrated that the biofloc culture system was associated with diverse bacterial communities. In this study, the overall predominant bacterial phyla across all samples were Cyanobacteria, Bacteroidetes, Proteobacteria and Planctomycetes. The health indicator communities such as Rhodobacteraceae and Flavobacteriaceae were enriched in the rearing pond water and Penaeus vannamei surface. The pond water and shrimp surface shared $>43 \%$ of the OTUs, which indicated that the pond water could have a significant influence on the shrimp surface microbiota. Considering the good health status of shrimp with $>80 \%$ survival rate in the biofloc system, further studies should be done to research shrimp immunity and disease resistance in the biofloc system.

\section{Acknowledgements}

This study was financially supported by the DBTINDO-UK BBSRC project (BT/IN/Indo-UK/BBSRCAqua/37/RJK/2015-16). The DBT Bioinformatics Centre, College of Fisheries, Mangalore is gratefully acknowledged for carrying out the sequence analysis work.

\section{References}

Bengtsson, M.M., Øvreås, L. 2010. Planctomycetes dominate biofilms on surfaces of the kelp Laminaria hyperborea. BMC Microbiology 10:261. https://doi.org/10.1186/1471-2180-10-261

Cardona, E., Gueguen, Y., Magré, K., Lorgeoux, B., Piquemal, D., Pierrat, F., Noguier, F., Saulnier, D. 2016. Bacterial community characterization of water and intestine of the shrimp Litopenaeus stylirostris in a biofloc system. BMC Microbiology 16:157. https://doi.org/10.1186/s12866-016-0770-z

Chao, A., 1984. Nonparametric estimation of the number of classes in a population. Scandinavian Journal of Statistics 11:265-270.

Colwell, R.K., Coddington, J.A. 1994. Estimating terrestrial biodiversity through extrapolation. Philosophical Transactions of the Royal Society B: Biological Sciences 345:101-118.

Dai, L., Wang, L., Sun, J., Zheng, L., Qi, B. 2018. Microbial community structure and diversity of shrimp paste at different fermentation stages. bioRxiv 334136. https://doi.org/10.1101/334136

Deng, Y., Xu, X., Yin, X., Lu, H., Chen, G., Yu, J., Ruan, Y. 2019. Effect of stock density on the microbial community in biofloc water and Pacific white shrimp (Litopenaeus vannamei) gut microbiota. Applied Microbiology and Biotechnology 103:4241-4252. https://doi.org /10.1007/s00253-019-09773-4

DeSantis, T.Z., Hugenholtz, P., Larsen, N., Rojas, M., Brodie, E.L., Keller, K., Huber, T., Dalevi, D., Hu, P., Andersen, G.L. 2006. Greengenes, a chimera-checked 16S rRNA gene database and workbench compatible with ARB. Applied and Environmental Microbiology
72:5069-5072. https://doi.org/10.1128/AEM.03006-05

Emerenciano, M., Cuzon, G., Paredes, A., Gaxiola, G. 2013. Evaluation of biofloc technology in pink shrimp Farfantepenaeus duorarum culture: growth performance, water quality, microorganisms profile and proximate analysis of biofloc. Aquaculture International 21:13811394. https://doi.org/10.1007/s10499-013-9640-y

Fan, J., Limei, C., Guoqin, M., Haoran, Z., Jinfang, Y., Deng, D., Yingfei, M., 2019. Dynamics of the gut microbiota in developmental stages of Litopenaeus vannamei reveal its association with body weight. Scientific Reports 9:734. https://doi.org/10.1038/s41598-018-37042$\underline{3}$

Hou, D., Huang, Z., Zeng, S., Liu, J., Wei, D., Deng, X., Weng, S., He, Z., $\mathrm{He}, \mathrm{J}$. 2017. Environmental factors shape water microbial community structure and function in shrimp cultural enclosure ecosystems. Frontiers in Microbiology 8:2359. https://doi.org/10.3389/fmicb .2017 .02359

Huerta-Rábago, J.A., Martínez-Porchas, M., Miranda-Baeza, A., NievesSoto, M., Rivas-Vega, M.E., Martínez-Córdova, L.R. 2019. Addition of commercial probiotic in a biofloc shrimp farm of Litopenaeus vannamei during the nursery phase: Effect on bacterial diversity using massive sequencing 16S rRNA. Aquaculture 502:91399. https://doi.org/10.1016/j.aquaculture.2018.12.055

Jost, L. 2007. Partitioning diversity into independent alpha and beta components. Ecology 88:2427-2439. https://doi.org/10.1890/06$\underline{1736.1}$

Krummenauer, D., Samocha, T., Poersch, L., Lara, G., Wasielesky, W. 2014 The reuse of water on the culture of pacific white shrimp, Litopenaeus vannamei, in BFT system. Journal of the World Aquaculture Society 45:3-14. https://doi.org/10.1111/jwas.12093

Langille, M.G., Zaneveld, J., Caporaso, J.G., McDonald, D., Knights, D., Reyes, J.A., Clemente, J.C., Burkepile, D.E., Vega Thurber, R.L., Knight, R., Beiko, R.G., Huttenhower, C. 2013. Predictive functional profiling of microbial communities using 16S rRNA marker gene sequences. Nature Biotechnology 31:814-821. https://doi.org 110.1038/nbt.2676

Office International des Épizooties (OIE). 2003. Manual of diagnostic tests for aquatic animals. $4^{\text {th }}$ Edition. OIE, Paris. $358 \mathrm{pp}$.

Oksanen, J., Blanchet, F.G., Friendly, M., Kindt, R., Legendre, P., McGlinn, D., Minchin, P.R., O'Hara, R.B., Simpson, G.L., Solymos, P. Stevens, M.H.H., Eduard Szoecs, E., Wagner, H. 2018. vegan: Community Ecology Package. $\mathrm{R}$ package version 2.5-2. https://CRAN.R-project.org/package=vegan

Parks, D.H., Tyson, G.W., Hugenholtz, P., Beiko, R.G. 2014. STAMP: statistical analysis of taxonomic and functional profiles. Bioinformatics 30:3123-3124. https://doi.org/10.1093/bioinformatics Ibtu494

Pilotto, M. R., Goncalves, A., Vieira, F.N., Seifert, W.O.., Bachère, E., Rosa, R.D., Perazzolo, L.M. 2018. Exploring the impact of the biofloc rearing system and an oral WSSV challenge on the intestinal bacteriome of Litopenaeus vannamei. Microorganisms 6:83. https: //doi.org/10.3390/microorganisms6030083

Rathgeber, C., Yurkova, N., Stackebrandt, E., Schumann, P., Beatty, J.T., Yurkov, V. 2005. Roseicyclus mahoneyensis gen. nov., sp. nov. an aerobic phototrophic bacterium isolated from a meromictic lake. International Journal of Systematic and Evolutionary Microbiology 55:1597-603. https://doi.org/10.1099/ijs.0.63195-0

Rego, M.A.S., Sabbag, O.J., Soares, R.B., Peixoto, S., 2018. Technical efficiency analysis of marine shrimp farming (Litopenaeus vannamei) in biofloc and conventional systems: A case study in north-eastern Brazil. Anais da Academia Brasileira de Ciências 90:3705-3716. http://dx.doi.org/10.1590/0001-3765201820170953

Schloss, P.D., Westcott, S.L., Ryabin, T., Hall, J.R., Hartmann, M., 
Hollister, E.B., Lesniewski, R.A., Oakley, B.B., Parks, D.H., Robinson, C.J., Sahl, J.W., Stres, B., Thallinger, G.G., Van Horn, D.J., Weber, C.F. 2009. Introducing Mothur: open-source, platform-independent, community-supported software for describing and comparing microbial communities. Applied and Environmental Microbiology 75:7537-7541. https://doi.org/10.1128/AEM.01541-09

Trappen, V.S., Mergaert, J., Swings, J. 2003. Flavobacterium gelidilacus sp. nov., isolated from microbial mats in Antarctic lakes. International Journal of Systematic and Evolutionary Microbiology 53:1241-1245. https://doi.org/10.1099/ijs.0.02583-0

Vikram, S., Guerrero, L.D., Makhalanyane, T.P., Le, P.T., Seely, M. Cowan, D.A. 2015. Metagenomic analysis provides insights into functional capacity in a hyperarid desert soil niche community. Environmental Microbiology 18:1875-1888. https://doi.org/10.1111 /1462-2920.13088

Vogan, C.L., Powell, A., Rowley, A.F. 2008. Shell disease in crustaceans - just chitin recycling gone wrong? Environmental Microbiology 10:826-835. https://doi.org/10.1111/j.1462-2920.2007.01514.X

Zheng, Y., Yu, M., Liu, J., Qiao, Y., Wang, L., Li, Z., Zhang, X.H., Yu, M. 2017. Bacterial community associated with healthy and diseased pacific white shrimp (Litopenaeus vannamei) larvae and rearing water across different growth stages. Frontiers in Microbiology 8:1362. https://doi.org/10.3389/fmicb.2017.01362 


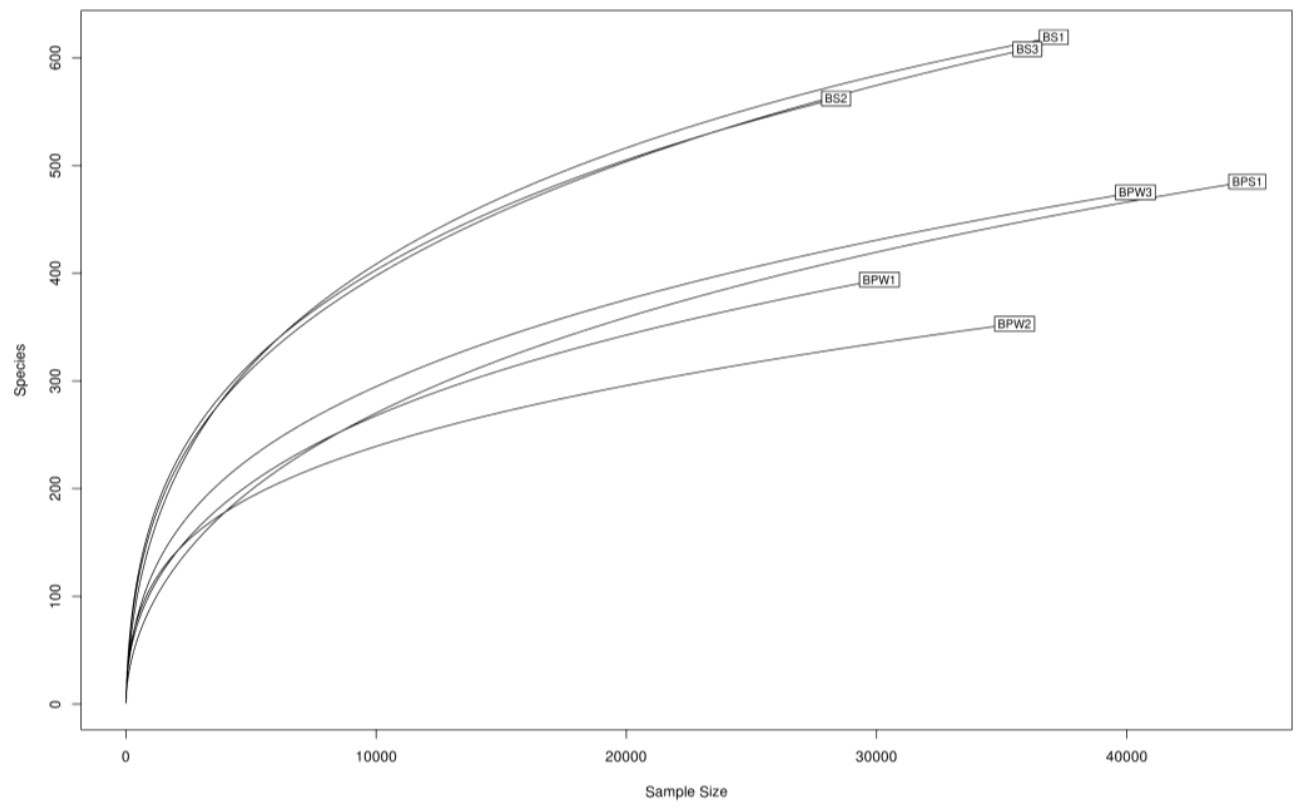

Supplementary Fig. 1. Rarefaction curves of the microbiota associated with the pond water as well as shrimp surface in a biofloc system. BPS1- Biofloc Prestocking Pond water; BPW1-Biofloc Pond Water (35dps); BPW2- Biofloc Pond Water (55dps); BPW3-Biofloc Pond water(70dps); BS1-BS3: Biofloc Shrimp surface at 35, 55 and 70 dps respectively.

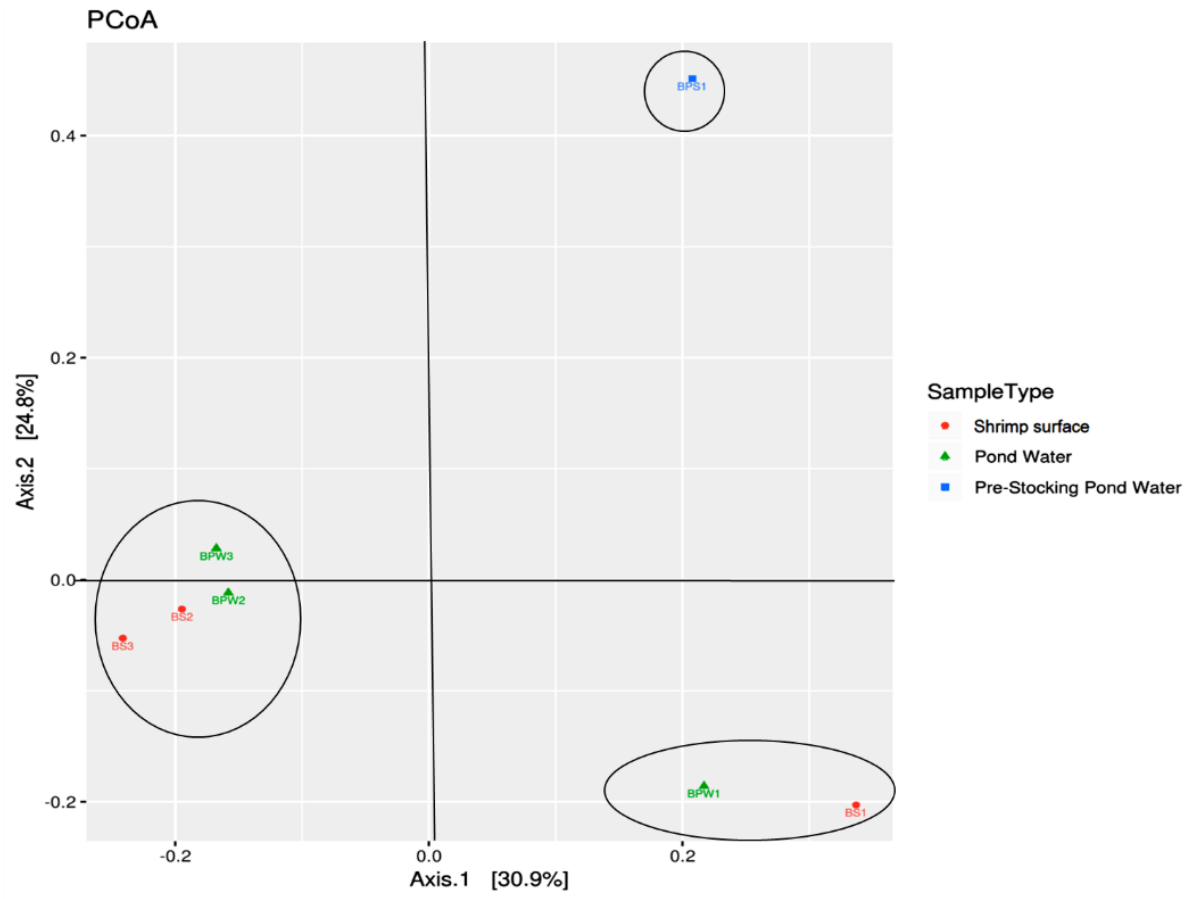

Supplementary Fig. 2. PCoA plot for Pond Water vs. Shrimp surface comparison. BPS1- Biofloc Prestocking Pond water; BPW1-Biofloc Pond Water (35dps); BPW2- Biofloc Pond Water (55dps); BPW3-Biofloc Pond water (70dps); BS1-BS3: Biofloc Shrimp surface at 35, 55 and 70 dps, respectively. 
Supplementary Table 1. Different Alpha diversity indices of which Chaol and ACE represent the richness of the sample and Shannon, Simpson, InvSimpson and Fisher represent both richness and relative abundance of bacteria in pond water as well as shrimp surface in a biofloc rearing system. BPS1- Biofloc Prestocking Pond water; BPW1-Biofloc Pond Water (35dps); BPW2Biofloc Pond Water (55dps); BPW3-Biofloc Pond water (70dps); BS1 -BS3: Biofloc Shrimp surface at 35, 55 and 70 dps respectively.

\begin{tabular}{lllllllll}
\hline Sample & Observed & Number of reads & Chao1 & ACE & Shannon & Simpson & Invimpson & Fisher \\
\hline BPS1 & 485 & 167448 & 676.838 & 692.814 & 2.922 & 0.853 & 6.819 & 76.003 \\
BPW1 & 394 & 123874 & 587.717 & 553.807 & 3.669 & 0.950 & 19.984 & 64.002 \\
BPW2 & 353 & 154676 & 519.528 & 503.957 & 3.862 & 0.963 & 26.949 & 54.458 \\
BPW3 & 475 & 178828 & 664.444 & 674.864 & 3.754 & 0.954 & 21.651 & 75.619 \\
BS1 & 619 & 157492 & 786.000 & 787.070 & 3.813 & 0.927 & 13.782 & 105.547 \\
BS2 & 562 & 133880 & 742.197 & 755.220 & 4.348 & 0.972 & 35.666 & 99.313 \\
BS3 & 608 & 163540 & 817.817 & 837.103 & 4.251 & 0.968 & 31.427 & 103.902 \\
\hline
\end{tabular}

